Revista de Comunicación y Salud, 2017, Vol. 7, pp. 187-198

Editado por Cátedra de Comunicación y Salud

ISSN: 2174-5323 (impreso) 2173-1675 (electrónico)

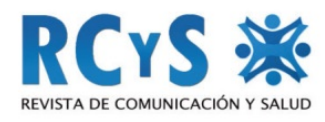

Enviado 28 de mayo de 2017

Aprobado 15 de julio 2017

\title{
APORTES PARA LA COMUNICACIÓN EN SALUD INTERCULTURAL: LA COPRODUCCIÓN DE CONOCIMIENTO EN DIABETES
}

\author{
Contributions for communication in intercultural health: co-production of \\ knowledge in diabetes
}

Soledad Rojas Rajs ${ }^{1}$

CONACYT - Universidad Autónoma Metropolitana. México

\begin{abstract}
Resumen
Las diferencias culturales y lingüísticas entre proveedores y usuarios de servicios sanitarios implican barreras para la comunicación en salud. Aunque es imprescindible sortear barreras lingüísticas y lograr aceptabilidad cultural de las comunicaciones, es igualmente importante considerar que tanto las relaciones interculturales como las sanitarias se caracterizan por ser asimétricas, y son por tanto relaciones de poder. La interculturalidad, idealmente, requiere ciertas condiciones de igualdad en las interacciones, que posibiliten diálogo, intercambio y aprendizaje a partir de racionalidades, tradiciones y valores entre distintos actores. Pero dado el carácter normativo y prescriptivo de la salud, la comunicación sanitaria puede reforzar asimetrías e impedir la disolución de barreras culturales. Un factor que sostiene las relaciones asimétricas entre proveedores de servicios de salud y usuarios, es que únicamente los primeros se reconocen como poseedores de conocimiento legítimo y científico. Experiencias exitosas en América Latina y Canadá han logrado integrar el conocimiento en salud de pueblos originarios a las prácticas institucionales. El reto es trasladar esta experiencia al ámbito de la comunicación. Este ensayo propone que la comunicación intercultural en salud puede enriquecerse a partir de procesos de co-producción de conocimiento de base comunitaria. Se reflexiona sobre algunas experiencias canadienses y latinoamericanas y un problema de salud concreto - la diabetes- para delinear algunas lecciones aprendidas sobre comunicación en salud intercultural que buscan aportar a la formación profesional de mediadores interculturales en comunicación sanitaria y a enriquecer la comunicación intercultural en salud, con base en producción conjunta de conocimiento.
\end{abstract}

Palabras clave: Salud intercultural; comunicación en salud intercultural; diabetes; coproducción de conocimiento en salud

\footnotetext{
${ }^{1}$ Autor de contacto: Soledad Rojas-Rajs srojasrajs@gmail.com
} 
Aportes para la comunicación en salud intercultural: la coproducción de conocimiento en diabetes

\begin{abstract}
Cultural and linguistic differences between providers and users of health services imply barriers to health communication. Although it is imperative to overcome linguistic barriers and to achieve cultural acceptability of communications, it is equally important to consider that both, intercultural and health relationships, are characterized by asymmetry and power relations. Interculturality, ideally, requires certain conditions of equality in the interactions, which enable dialogue, exchange and learning from rationalities, traditions and values between different actors. But healthcare's nature is normative and prescriptive. Health communication could be reinforce asymmetries and difficult the dissolution of cultural barriers. One factor that support the asymmetric relationships between health service providers and users is that only the former are recognized as having legitimate and scientific knowledge. Successful experiences in Latin America and Canada have succeeded in integrating the health knowledge of indigenous peoples into institutional practices. The challenge is to transfer this experience to the field of communication. This essay proposes that intercultural health communication can be improvment by processes of co-production of knowledge, community-based. Thinking about some Canadian and Latin American experiences and a specific health problem - diabetes - there are some lessons learned about intercultural health communication that can contribute to the professional training of intercultural mediators in health communication and to enrich intercultural health communication, based in coproduction of knowledge.

Keywords: Intercultural Health; Intercultural Health Communication; Diabetes; Coproduction of Knowledge

Para citar el artículo

Rojas Rajs, S. (2017). Aportes para la comunicación en salud intercultural: la coproducción de conocimiento en diabetes. Revista de Comunicación y Salud, vol. 7, 187-198.

Recuperado

http://revistadecomunicacionysalud.org/index.php/rcys/article/view/124
\end{abstract}


Aportes para la comunicación en salud intercultural: la coproducción de conocimiento en diabetes

\section{PROBLEMAS DE COMUNICACIÓN INTERCULTURAL EN SALUD}

Los problemas para lograr comunicación en salud efectiva, a causa de diferencias culturales y lingüísticas entre el personal de salud y los usuarios de los servicios sanitarios se han identificado desde hace tiempo como barreras para alcanzar logros en salud (Alarcón M, Vidal H, \& Rozas, 2003). Sin embargo, los esfuerzos por mitigar estas dificultades a menudos son de carácter instrumental, más que derivados de una visión intercultural sobre comunicación y salud. Aunque el término "intercultural" está presente en muchos programas de salud dirigidos a pueblos originarios en países de América Latina y es un ideal a conseguir, en la práctica han sido más bien escasos los esfuerzos por reconocer necesidades culturales y locales especificas, sobre todo si entran en contradicción con la visión de cuáles son las prescripciones sanitarias correctas. En el caso de México, no se ha impulsado institucionalmente, desde el sector salud, la integración de mediadores interculturales o intérpretes que formen parte del personal de salud, lo que se observa en la inexistencia de puestos específicos para profesiones emergentes, como las nuevas licenciaturas en salud intercultural, promoción de la salud, educación para la salud que han aparecido en la última década en algunas universidades mexicanas. Incluso es limitado, a nivel nacional, el fomento a la educación cultural y capacitación lingüística del personal de salud de las instituciones públicas. En parte, esto deriva de la ausencia de un enfoque intercultural o una definición limitada de lo que significa enmarcar las acciones de salud en una perspectiva interculturalidad.

En este trabajo el planteamiento inicial es que para realizar comunicación en salud intercultural, este enfoque debe orientar desde el nivel de la planificación y generación de procesos de comunicación en salud, hasta la ejecución concreta y particular, materializada en estrategias y acciones comunicativas (Rizo García, 2013). Por ello, se sostiene que no basta con asegurar el acceso lingüístico o el "ajuste" de los mensajes de salud a los entornos locales, en busca de su aceptabilidad cultural. Aunque es imprescindible trabajar la interculturalidad desde la dimensión lingüística y con profundo conocimiento de los códigos culturales locales, es igualmente importante considerar que las relaciones interculturales y sanitarias acontecen en el marco de relaciones de poder. En este sentido, se reconoce que existe una correlación de fuerzas asimétrica entre actores de culturas y territorios diferentes, en tanto que hay marcos culturales hegemónicos (Alarcón M et al., 2003; Ávila Romero, 2011; Rizo García, 2013) y en el caso de la salud, medicina hegemónica (Briggs, 2005; Menéndez, 2004; Navarro, 1998). Si esto no es tomado en cuenta por quienes planifican y desarrollan estrategias de comunicación en salud interculturales, estas podrían -en el caso más extremogenerar efectos adversos, en vez de apoyar la consecución de logros en salud, puesto que a través de la comunicación pueden reproducirse estereotipos, mandatos y jerarquías sociales (Briggs, 2005) que fomentan las desigualdades en salud. Se corre el riesgo de fungir como traductor y no como mediador intercultural.

Aunque no es posible construir comunicación en salud desde un escenario totalmente horizontal, la perspectiva intercultural requiere un esfuerzo específico para lograr las mejores condiciones de igualdad en las interacciones entre actores. Es decir, se trata de nivelar, en la medida de lo posible (casi siempre lo posible es mucho más de

Revista de Comunicación y Salud, 2017, Vol. 7, pp. 187-198 
Aportes para la comunicación en salud intercultural: la coproducción de conocimiento en diabetes

lo que se hace), la posición relativa de los actores en el campo, pues sólo así se puede lograr que los procesos dialógicos y de aprendizaje tengan un valor relativamente similar, o suficiente al menos, para que se produzcan intercambios de conocimientos y experiencias que puedan ser integrados a las prácticas en salud, tanto institucionales, como colectivas.

Con este marco, se plantean cinco lecciones aprendidas sobre comunicación en salud intercultural, en experiencias latinoamericanas y canadienses, que por la extensión del ensayo solo se refieren, para consulta posterior. El documento se estructura en en torno a estas cinco lecciones, partiendo de la necesidad de darle un lugar privilegiado al vínculo entre interculturalidad y comunicación; abordando posteriormente la cuestión del conocimiento en salud y hablando también de un problema de salud específico: la diabetes.

Finalmente se plantea que la colaboración intercultural en la producción de conocimiento no está exenta de conflictos y dificultades (Mato, 2008), sin embargo, es una ruta fértil que vale la pena transitar porque enriquece la planificación de estrategias de comunicación sanitaria y promueve la apropiación social del conocimiento en salud, en un sentido amplio.

\section{Elaborar materiales de comunicación no significa comunicar}

A fines de la década de los noventa comencé a realizar investigación para la comunicación en el sector salud en México, inicialmente como consultora del sector privado. Una buena parte de los proyectos que realicé consistían en probar campañas de comunicación en salud aún no implementadas (pre-test) pero en otros casos afortunadamente, los menos- se trataba de probar las campañas ya implementadas (test). Sobra decir que en la mayoría de las ocasiones, las campañas resultaban reprobadas por sus públicos, pues no habían sido concebidas con base en las comunidades receptoras: no se comprendían, no utilizaban elementos propios de la vida cotidiana de los destinatarios, existían enormes brechas lingüísticas y a veces, estaban en franca contradicción con las lógicas y dinámicas locales. En vez de generar adherencia a tratamientos $\mathrm{y} / \mathrm{o}$ conductas, producían rechazo, desconfianza y distanciamiento. Al menos dos de estas campañas fallidas, en mi experiencia profesional, fueron implementadas a nivel nacional por las principales instituciones públicas de salud de México.

¿Qué sucedió? Que se construyeron campañas con pretensiones universales, desde el nivel central y se distribuyeron con el modelo de cascada, es decir, pensando que un solo ejercicio comunicativo era suficiente para atender la diversidad, aun tomando en cuenta que estaban dirigidas a ciertos nichos, en teoría definidos: "las mujeres", "las gestantes", "las comunidades rurales", por ejemplo. ¿Lograron comunicar? Pobremente. Esto es porque la comunicación en salud no puede concebirse sólo como piezas, sino como un proceso que es resultado de las interacciones entre emisores y destinatarios, por lo tanto requiere una estrategia que anticipe cómo serán esas interacciones, entre quienes ocurrirán y desde qué comunidades socioculturales, en qué condiciones de vida. Al igual que pasa con una perspectiva intercultural, la comunicación en salud 
Aportes para la comunicación en salud intercultural: la coproducción de conocimiento en diabetes

requiere construirse pensando en dónde estarán situados los receptores y cómo serán los procesos de comunicación. En ese sentido, interculturalidad y comunicación tienen muchos puntos en común: el enfoque en las interacciones, el intercambio y la matriz cultural común que permite compartir significados (Rizo García, 2013). Nunca deben elaborarse materiales de comunicación en salud sin considerar estas tres dimensiones ¿Quiénes son los actores de las interacciones? ¿Cómo puede promoverse el intercambio? ¿Qué matrices socioculturales requieren dialogar?

\section{La interculturalidad requiere ser una perspectiva universal en comunicación sanitaria}

Sosteniendo el rechazo a un conocimiento único en salud, que podría no tener pertinencia sociocultural (Mato, 2008; Rizo García, 2013), se puede proponer un juego inverso: si algo debe ser universal, eso sería la interculturalidad (Verdugo Urrejola et al., 2008). Si se concibe como un enfoque dialógico, que implica reconocer al otro y sus diferencias respecto a nosotros mismos, es una perspectiva que puede aplicarse a la comunicación sanitaria dirigida a cualquier sociedad o colectivo humano, pues todos somos portadores de cultura.

La comunicación sanitaria siempre requiere tomar en consideración el contexto cultural en el que va a implementarse. Sin embargo, como bien han aprendido ya en Guatemala los implementadores del modelo de Salud Incluyente (Verdugo Urrejola et al., 2008), pueden existir visiones limitadas sobre salud intercultural. Por ejemplo, restringida a contextos de población indígena, por lo tanto se presupone que la perspectiva no sería aplicable en otros contextos, aun cuando todo contexto es sociocultural. Otro problema para concebir la interculturalidad es concebir las tradiciones y costumbres, recursos, terapias, cosmogonía, como si estuvieran en estado puro y no hubieran realizado ya permutas y transacciones simbólicas y prácticas en el campo de la salud. Sin embargo, las lógicas sociales y culturales han sido mediadas y modeladas por otros elementos culturales con los que históricamente han tenido contacto, incluida la relación con las instituciones de salud, los gremios médico y de enfermería, las políticas sanitarias en el país. Por ello es siempre necesario actualizar el conocimiento sobre su estado y entender sus procesos de hibridación.

Además, el proceso salud enfermedad-atención, aun sí lo enfocásemos desde su concepción occidental, está relacionado con la cultura:

"Desde el punto de vista familiar y comunitario, las formas de autoatención, organización y acción de los grupos comunitarios son expresión de las lógicas socioculturales de esos colectivos. Al realizar análisis y acciones guiadas por la interculturalidad, se encontrará una red imbricada de conceptos y formas de enfrentar la enfermedad y la muerte, emanadas de la situación de clase, la actividad económica, las condiciones de vida, la religión, el status, los aspectos genéricos, la experiencias familiares y locales, entre muchos otros, todas y todos relacionados con diferentes matrices socioculturales. En síntesis, la interculturalidad es una perspectiva universal aplicable a cualquier contexto social y toda acción humana" (Verdugo Urrejola et al., 2008, p. 12). 
Aportes para la comunicación en salud intercultural: la coproducción de conocimiento en diabetes

En este sentido, cualquier problema de salud que se analice desde una perspectiva intercultural, desde la atención del parto y la mortalidad materna, hasta las enfermedades crónicas, como la diabetes, podrá incluir la dimensión sociocultural en su interpretación del proceso salud-enfermedad, entendiendo tanto los riesgos asociados a las características y condiciones de vida de los colectivos, como los vínculos, fuerzas y capacidades que los grupos humanos construyen para responder a sus problemas de salud. Un comunicador intercultural requiere entonces construir siempre una mirada amplia hacia las lógicas colectivas e individuales, que deben nutrir la comunicación estratégica.

\section{El conocimiento en salud es un eje de poder para nivelar las relaciones entre actores}

El conocimiento en salud es uno de los puntos clave para la comunicación en salud porque ser uno de los ejes de poder en las relaciones interculturales en el campo sanitario. Como campo disciplinario, la medicina y las ciencias de la salud se han construido con base en la argumentación de que existen sólo algunos conocimientos valiosos: aquellos que están científicamente validados. Sin embargo, la idea de una ciencia infalible, única y universal es limitativa para los procesos humanos de aprendizaje (Mato, 2008) y es ciertamente incompatible con una perspectiva intercultural.

Un ejemplo de esto es el enfoque de la prevención de la diabetes, actualmente concentrada en promover estilos de vida saludables, particularmente respecto a alimentación. Esta perspectiva presupone que los individuos tienen siempre y total autonomía para tomar decisiones y optar por conductas y prácticas de vida saludables; y además, que los cambios de actitud, por sí mismos tienen un efecto significativo en la salud, independientemente de otros factores (Carvalho, 2008), incluidas las condiciones económicas y sociales. Pero lo más importante es que el concepto estilos de vida saludables está planteado en el ámbito individual, sin considerar que incluso las conductas individuales, responden a factores sociales (Benach y Muntaner, 2008) y que la vida social es diversa culturalmente. No todas las soluciones funcionan para cualquier comunidad de sentido.

Además de la disputa cognitiva, en términos de cómo atender y resolver la diabetes, otro rasgo hegemónico basado en el conocimiento suele ser parte de la comunicación en salud: la comunicación en salud es generalmente vertical y lineal, y destaca las relaciones jerárquicas. Podríamos describir el modelo de la siguiente manera: el conocimiento es creado por los expertos, en espacios autorizados para producir conocimiento sobre salud, legitimidad que se consigue con entrenamiento especializado, disponibilidad de tecnologías y autoridad institucional. En segundo lugar, el conocimiento debe traducirse a lenguajes no técnicos y accesibles para los legos. En tercer lugar, el conocimiento debe ser difundido a través de distintos medios y canales. Finalmente, el público recibe el conocimiento, y al asimilarlo cognitivamente, se apropia de él, consiguiendo una "permuta cognitiva" que se presupone se reflejará en su comportamiento (Briggs, 2005) En la verticalidad de este modelo se manifiestan las relaciones de poder entre los emisores y los destinatarios. En la práctica, ambas situaciones se entrelazan: las relaciones asimétricas comunicativas (entre emisores y 
Aportes para la comunicación en salud intercultural: la coproducción de conocimiento en diabetes

receptores) y cognitivas (entre expertos y legos), están presentes en la comunicación sanitaria y ello impide un verdadero ejercicio intercultural.

El reto de nivelar la posición relativa de los actores es complejo, pero no es insalvable. Los límites son la creatividad de los comunicadores interculturales y los profesionales de la salud, trabajando en conjunto con las comunidades y colectivos interesados. Una estrategia que ha resultado muy exitosa en el modelo de salud incluyente, tanto en Bolivia como en Guatemala, ha sido la elaboración de estrategias de comunicación intercultural para el personal de salud y no solamente para las comunidades, o promotores de salud comunitarios. Los manuales de referencia elaborados en Bolivia (Gobierno Autonomo Departamental de la Paz/Servicio Departamental de Salud La Paz, 2014) enseñan fundamentalmente al personal de salud no familiarizado con los detalles del sistema tradicional de atención cuáles son los síntomas de lo que han denominado "enfermedades socioculturales" y quién debe ser el tratante, otorgando con este manual un estatus diferenciado a curanderos tradicionales, parteras, herbolarios y hueseros frente al personal de salud profesionalizado. Estos manuales, conforman un proceso comunicativo muy relevante: transforman el horizonte de sentido del personal de salud, y reduce las barreras interpretativas sobre los padecimientos que refieren las comunidades en las que trabajan.

Existe también un manual para referir al primer nivel de atención o segundo, sobre todo en caso de emergencias. Ambos constituyen una muestra de qué puede hacer la comunicación por construir un horizonte más igualitario, aunque se restringa el ámbito de la toma de decisiones en salud.

\section{Los problemas de salud expresan las relaciones socioculturales: el caso de la diabetes}

Desde una perspectiva intercultural amplia se considera que los problemas de salud están social y culturalmente determinados. La diabetes, por ejemplo, es un grave problema de salud en México y el mundo, con particularidades para las comunidades indígenas y rurales.

Analizar la diabetes es pertinente por varios motivos: a) porque como problema de salud ejemplifica bien la influencia social y cultural en los procesos de saludenfermedad; b) porque existe un conocimiento científico legitimado que promueve un solo tipo de abordaje para este problema de salud, en el ámbito preventivo y de la atención primaria en salud y c) porque es una de las enfermedades que se busca atender con una solución universal, en general, a través del enfoque de estilos de vida saludables (EVS), un enfoque problemático porque difícilmente podemos hablar de EVS culturalmente homogéneos. Otro motivo para elegir este tema, es porque gran parte de la producción académica y científica sobre interculturalidad en salud está centrada en la atención del embarazo y el parto en pueblos originarios, que son temas muy relevantes pero no deben conformarse como los únicos problemas de interés para la comunicación en salud intercultural.

La diabetes mellitus, especialmente la tipo 2, es actualmente uno de los problemas de salud más graves a nivel mundial. En México, la DM2 es hoy la segunda causa de

Revista de Comunicación y Salud, 2017, Vol. 7, pp. 187-198 
Aportes para la comunicación en salud intercultural: la coproducción de conocimiento en diabetes

muerte y muestra una tendencia creciente de población afectada. Entre 2000 y 2012 el número de personas diagnosticadas como diabéticas se duplicó, alcanzando al $9.2 \%$ de la población adulta; adicionalmente se estima que existe una proporción similar de enfermos no diagnosticados (Hernández-Ávila, 2012). La mortalidad por diabetes en México es una de las más altas del mundo (World Health Organization, 2016). A pesar de las diversas acciones encaminadas a reducir la incidencia de diabetes, incluida la promoción de EVS en la comunicación, las proyecciones a futuro muestran un incremento exponencial de casos. Una mayor prevalencia de diabetes en México está asociada a condiciones de vida desventajosas (Medina Gómez \& López Arellano, 2010), y menor acceso a alimentos sanos, espacios recreativos para actividad física. Por otro lado, en zonas de muy alta prevalencia de diabetes, en general hay presencia de riesgos asociados, como disruptores endocrinos y otros tóxicos (Aguilar Salinas, Hernández Jiménez, Hernández Ávila, \& Hernández Ávila, 2015; Ruiz-Ramos, Calderón-Garcidueñas, López-Amador, Melo-Santiesteban, \& Waliszewski-Kubiak, 2014).

¿Cuáles son las estrategias de comunicación sanitaria intercultural sobre diabetes en México? Lamentablemente, no existen como tal (Rojas-Rajs, 2016) y cuando se lanzan algunas piezas de comunicación con algunas adecuaciones culturales, no se tratan de esfuerzos sostenidos, es decir, no se conforman como estrategias en el tiempo. Un vistazo a los recursos gráficos y comunicativos más frecuentes ubican a la diabetes siempre en contextos urbanos, siempre asociada con la obesidad y siempre asociada a la representación mediática de la comida chatarra, que no muestra el impacto del cambio en la cultura alimentaria. Un estudio sobre prevención intercultural de la diabetes en el pueblo Pemón Kamarakoto en Venezuela mostró las dificultades para comunicar desde la perspectiva hegemónica de la medicina, puesto que en las comunidades visitadas en esa investigación antropológica la mayoría de la población diabética no tenía sobrepeso (Bautista, 2014) y las recomendaciones sobre alimentación y obesidad no hacían sentido. Se trata de poblaciones con gran predisposición genética frente a la enfermedad, lo que desgraciadamente ha resultado ser un rasgo compartido entre diversos pueblos originarios de América y Asia principalmente. Otras imágenes y campañas proponen actividad física con equipamiento urbano e incluyen innumerables referentes que pueden ser totalmente desconocidos en diversas zonas rurales o de población que mayoritariamente habla un idioma indígena.

Además de estas cuestiones, un asunto que agrega complejidad es la necesidad de lograr cierta autonomía frente al conocimiento dominante al desarrollar una estrategia de comunicación. Por ejemplo, desde la epistemología biomédica la etiología de la diabetes es atribuible a causas cercanas de las enfermedades, por ejemplo las de origen genético y hereditario, las elecciones personales o los servicios de salud (Benach, 2012). El conocimiento sobre los contaminantes y otros factores que influyen en la diabetes, no suele recuperarse en la comunicación en salud y su exclusión de las comunicaciones modela una cierta visión social de la enfermedad: al declarar que depende mayoritariamente del estilo de vida individual, la comunicación produce y reproduce una hegemonía cognitiva sobre la enfermedad, que refuerza la idea de que 
Aportes para la comunicación en salud intercultural: la coproducción de conocimiento en diabetes

la acción individual es la más efectiva para combatir la diabetes, o la única posible, cuando las soluciones colectivas en salud son más duraderas y hay más adherencia a los tratamientos y recomendaciones. En el caso de las cosmovisiones de pueblos originarios, la noción de la colectividad suele ser un eje de la identidad cultural, por lo que seguir una estrategia que individualiza mensajes y responsabilidades, impide aprovechar las ventajas culturales de comunidades indígenas y otras comunidades organizadas en torno a una identidad común.

\section{La co-producción de conocimiento en diabetes como proceso de comunicación y el papel mediador de los comunicadores interculturales}

Dado que la comunicación en salud intercultural requiere de procesos participativos, que permitan conocer e interpretar a los otros, se ha considerado dentro de los procesos participativos la puesta en marcha de mecanismos que permitan la coproducción de conocimiento en salud (Rycroft-Malone et al., 2016). Este concepto, de gran arraigo en Canadá en los últimos 15 años, implica una reflexión constante sobre cómo lograr una mayor participación de quienes están en situación de subordinación cultural e institucional respecto al conocimiento en salud. Implica, entonces, dar a las comunidades el poder de participar, pero no sólo para evaluar u opinar sobre la pertinencia cultural, sino para generar conocimientos y contenidos para la comunicación en salud.

El reconocimiento de la diversidad cultural a través de la recuperación de conocimientos no es solo un aporte para los sistemas de salud. En Canadá, se ha definido como una deuda pendiente con los pueblos originarios (First Nation), que históricamente han sufrido desventajas sociales, discriminación y despojo (Canadian Institutes of Health Research \& Institute of Health Economics, 2011). Esto aplica también para América Latina y el resto del mundo, pues la condición desventajosa de la población indígena y la población rural, es una constante en la actualidad. En ese sentido, además de elaborar materiales específicos de comunicación en salud para los destinatarios hegemónicos, como el ejemplo de Bolivia, es necesario darle voz y vehículos de difusión a los contenidos construidos con base comunitaria, que revelan las formas de atención comunitaria, las dinámicas y también, plantean las necesidades en salud de los colectivos. El término que se utiliza para referirse a la participación en la construcción colectiva del conocimiento es "con base en la comunidad": Se trata así, de los modelos y metodologías de conocimiento basado en la comunidad y de la investigación participativa basada en la comunidad.

Los procesos de construcción colectiva de conocimiento tienen dificultades, en ellos se expresan también las relaciones de poder y contradicciones entre distintos miembros de las comunidades participantes (Mato, 2008; Rycroft-Malone et al., 2016). Un punto de partida utilizado tanto en experiencias canadienses como latinoamericanas, ha sido la planificación de actividades de contacto e intercambio para nutrir el conocimiento de los comunicadores. Se trata pues de establecer diálogos horizontales con profesionales de la salud de distintos orígenes: tanto aquellos que provienen de una formación más 
Aportes para la comunicación en salud intercultural: la coproducción de conocimiento en diabetes

hegemónica e institucional, como aquellos formados en contacto con distintas comunidades culturales; y a sanadoras y sanadores de base comunitaria (como parteras, hueseros, curanderos, etc.). Reunirlos y establecer un diálogo informado, respetuoso y abierto, puede ser un ejercicio de gran valor para identificar ejes estratégicos de la comunicación intercultural. Además, coloca a los distintos profesionales de la salud en un nivel relativamente igualitario, en tanto convoca con la misma voz y voto a personas que socialmente están en categorías distintas. Un paso subsecuente es la participación amplia de todos los interesados en las cuestiones de salud.

Ese tipo de procesos favorecen la planeación posterior de las comunicaciones y abre la puerta a un asunto de gran relevancia: ¿cuáles deben ser las competencias profesionales de los comunicadores interculturales, más allá de los conocimientos técnicos sobre producción de comunicación? Desde mi punto de vista, requieren desarrollar una alta sensibilidad para registrar información y formarse en técnicas de investigación cualitativas, de corte social y antropológico, para desarrollar mejor su papel de mediadores culturales. Estas habilidades técnicas y culturales permiten que la comunicadora o el comunicador puedan desarrollar estrategias de comunicación, que idealmente contarán con la retroalimentación de un equipo interdisciplinario en salud, pero que sobre todo requieren ser construidas con la participación de las comunidades destinatarias 0 beneficiarias. Esta formación permite sensibilizarse acerca de las necesidades de salud, las especificidades culturales, las barreras de los distintos actores y las características de las interacciones.

Ejemplo de ello, es que partir de los procesos participativos se han logrado estrategias importantes sobre diabetes entre los Inuit en Canadá (Canadian Institutes of Health Research \& Institute of Health Economics, 2011), que implican procesos tan diversos como la formación de grupos musicales, comunidades deportivas, colectivos de diversas edades, puesto que en todas esas interacciones se generan procesos comunicativos y de producción local de conocimientos. Si bien aún hay un largo camino por recorrer para que desde los bastiones más cerrados de la ciencia se reconozca y admita la validez del conocimiento tradicional y originario, la sistematización de las experiencias interculturales es un buen punto de partida.

\section{REFLEXIONES FINALES}

A modo de reflexión final, hay que agregar que cuando hablamos de comunicación en salud es importante plantear algunas consideraciones éticas. Primero, que hay que integrar a la concepción intercultural de la comunicación la perspectiva de género, para no reproducir estereotipos que favorezcan el rol subordinado de las mujeres en la sociedad, puesto que afecta la salud de las mujeres. Igual de relevante es respetar en todo momento el derecho a una vida libre de discriminación; y el derecho a la salud, que no debiese depender de nuestras condiciones, identidades o contextos socioculturales, ni de nuestro estado de salud. En ese sentido, hacer comunicación que avergüence o culpabilice a las personas por su mala situación sanitaria, es un terreno resbaloso, que debería ser evitado. No existe argumento intercultural que avale la 
Aportes para la comunicación en salud intercultural: la coproducción de conocimiento en diabetes

transgresión ética en la comunicación sanitaria. El compromiso intercultural ante todo es el reconocimiento y el respeto del otro y la comunicación no debe ser una excepción.

Así, se configura como reto para los comunicadores lograr la integración de estos saberes y habilidades. Con el objetivo de producir conocimiento conjunto, de base comunitaria, para orientar las prácticas interculturales de comunicación sanitaria, se puede promover la formación de mediadores interculturales.

\section{REFERENCIAS BIBLIOGRÁFICAS}

Aguilar Salinas, C. A., Hernández Jiménez, S., Hernández Ávila, M., \& Hernández Ávila, J. E. (2015). Acciones para enfrentar a la Diabetes. Documento de postura. México: Academia Nacional de Medicina.

Alarcón M, A. M., Vidal H, A., \& Rozas, J. N. (2003). Salud intercultural: Elementos para la construcción de sus bases conceptuales. Revista Medica de Chile, 131(9), 1061-1065. doi:http://doi.org/10.4067/S0034-98872003000900014

Ávila Romero, A. (2011). Universidades interculturales y colonialidad del saber. Revista de Educación y desarrollo, 16, 20-25.

Bautista, F. (2014). Prevención intercultural de la diabetes tipo II en pueblo Pemon Kamarakoto. Boletín Antropológico, 32(87), 28-44.

Benach, J., \& Muntaner, C. (2008). Desigualdades en salud: una epidemia que podemos evitar. Entrevista por Salvador López Arnal. Rebelión, 1-10.

Briggs, C. L. (2005). Perspectivas críticas de salud y hegemonía comunicativa: aperturas progresistas, enlaces letales. Revista de Antropología Social, 14, 101124.

Canadian Institutes of Health Research, \& Institute of Health Economics. (2011). Aboriginal Peoples 'Wellness in Canada: Scaling Up the Knowledge. Cultural Context and Community Aspirations. Report from the Roundtable. Alberta.

Carvalho, S. R. (2008). Promoción de la Salud, "empowerment" y educación: Una reflexión crítica como contribución a la reforma sanitaria. Salud Colectiva, 4(3), 335-347.

Gobierno Autonomo Departamental de la Paz/Servicio Departamental de Salud La Paz. (2014). Manual para la referencia de enfermedades socioculturales a la medicina tradicional. La Paz, Bolivia.

Hernández-Ávila, M. (2012). Evidencia para la política pública en salud en Diabetes mellitus: la urgencia de reforzar la respuesta en políticas públicas para su prevención y control. Ensanut.

Mato, D. (2008). No hay saber "universal", la colaboración intercultural es imprescindible*. Alteridades, 18(35), 101-116.

Medina Gómez, O. S., \& López Arellano, O. (2010). Una aproximación a los determinantes sociales de la diabetes mellitus tipo 2 en México. In M. del C. Chapela (Ed.), En el debate: la diabetes en México (pp. 25-52). México: Universidad Autónoma Metropolitana Xochimilco-División de Ciencias Biológicas y de la Salud.

Menéndez, E. L. (2004). Modelos de atención de los padecimientos: de exclusiones teóricas y articulaciones prácticas. En H. Spinelli (Ed.), Salud Colectiva. Cultura, 
Aportes para la comunicación en salud intercultural: la coproducción de conocimiento en diabetes

Instituciones y Subjetividad (pp. 11-48). Buenos Aires: Lugar Editorial.

Navarro, V. (1998). Concepto actual de salud pública. En Salud Pública (pp. 49-54). Madrid: McGraw Hill. Recuperado de http://uiip.facmed.unam.mx/deptos/salud/censenanza/spi/fundamentos/navarro.pdf

Rizo García, M. (2013). Comunicación E Interculturalidad. Reflexiones En Torno a Una Relación Indisoluble. Global Media Journal México Número, 10(19), 26-42.

Rojas-Rajs, S. (2016). La diabetes en la publicidad oficial para televisión mexicana: problemas y límites para la comunicación en salud. Salud Problema, 10(19), 9-21.

Ruiz-Ramos, R., Calderón-Garcidueñas, A., López-Amador, N., Melo-Santiesteban, G., \& Waliszewski-Kubiak, S. (2014). Exposición a arsénico y patologías asociadas. Revista Mexicana de Medicina Forense y Patología, 1(1), 14-23.

Rycroft-Malone, J., Burton, C. R., Bucknall, T., Graham, I. D., Hutchinson, A. M., \& Stacey, D. (2016). Collaboration and Co-Production of Knowledge in Healthcare: Opportunities and Challenges. Kerman University of Medical Sciences, 5(4), $221-$ 223. http://doi.org/10.15171/ijhpm.2016.08

Verdugo Urrejola, J. C., Hernández Mack, L., Morales, L. C., Herrera, V. O., Arriaga, C. A., \& Sánchez, C. (2008). Del dicho al hecho... Los avances de un primer nivel de atención en salud incluyente. Guatemala.

World Health Organization. (2016). Global Report on Diabetes. http://doi.org/ISBN978 9241565257 